Network Working Group

Request for Comments: 1137

Updates: RFC 976
S. Kille

University College London

December 1989

\title{
Mapping Between Full RFC 822 and RFC 822 with \\ Restricted Encoding
}

Status of this Memo

This RFC suggests an electronic mail protocol mapping for the Internet community and UK Academic Community, and requests discussion and suggestions for improvements. This memo does not specify an Internet standard. Distribution of this memo is unlimited.

This document describes a set of address mappings which will enable interworking between systems operating RFC 822 protocols in a general manner, and those environments where transfer of RFC 822 messages restricts the character set which can be used in addresses. UUCP transfer of RFC 822 messages is an important case of this [Crocker82a, Horton86a].

Specification

This document specifies a mapping between two protocols. This specification should be used when this mapping is performed on the Internet or in the UK Academic Community. This specification may be modified in the light of implementation experience, but no substantial changes are expected.

1. Introduction

Some mail networks which use RFC 822 cannot support the full character set required by all aspects of RFC 822. This document describes a symmetrical mapping between full RFC 822 addressing, and a form for use on these networks. Any addresses within the networks will not use the full RFC 822 addressing, and so any addresses encoded according to this standard will always represent remote addresses. This document derives from a mapping originally specified in RFC 987 [Kille86a], where the domain of application was more restricted. Two terms are now defined:

Full RFC 822

This implies full support for transfer to and from any legal RFC 822 address. In particular, the quoted-string form of local-part must be supported (e.g., <"Joe Soap"@foo.bar>). 
Restricted RFC 822

This implies a subset of RFC 822 addressing. The quoted-string form of local-part need not be supported. Standard UUCP mail transfer falls into this category. Restricted RFC 822 is undesirable, but in practice it exists in many places.

When a message is transferred from full RFC 822 to restricted RFC 822, and address forms used in full RFC 822 are involved, message loss may occur (e.g., it may not be possible to return an error message). This RFC describes a quoting mechanism which may be used to map between full RFC 822 and restricted RFC 822, in order to alleviate this problem.

2. Encoding

The RFC 822 EBNF meta notation is used. Any EBNF definitions taken from RFC 822 are prefixed by the string "822.".

The following EBNF is specified.

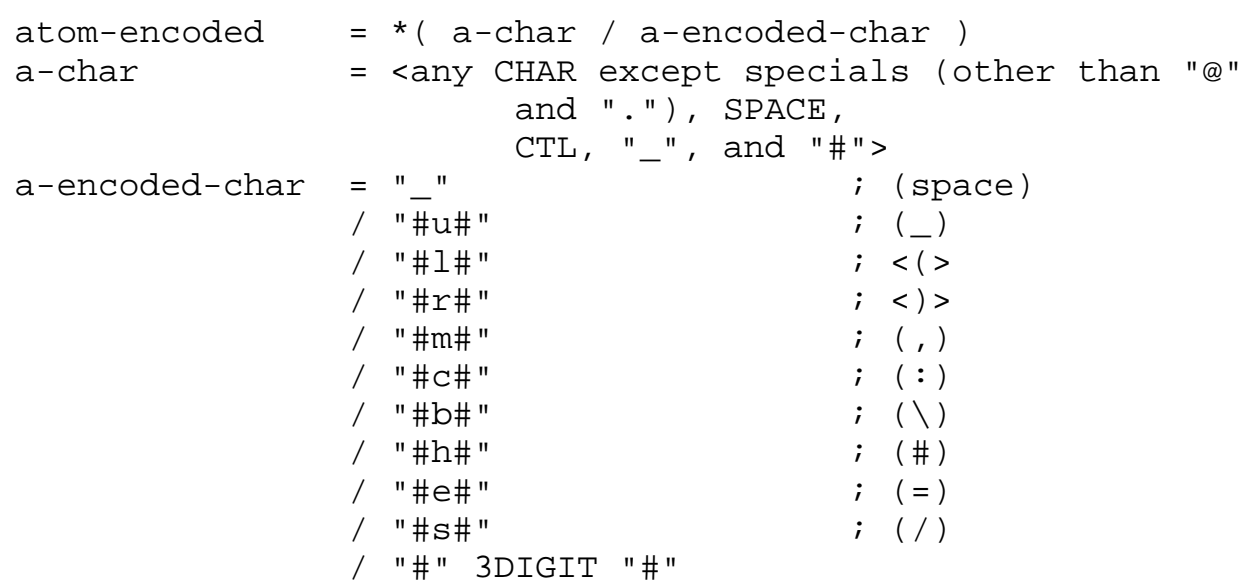

The 822.3DIGIT in EBNF.a-encoded-char must have range 0-127, and is interpreted in decimal as the corresponding ASCII character. The choice of special abbreviations (as opposed to decimal encoding) provided is based on the manner in which this mapping is most frequently used. There are special encodings for each of the Printablestring characters not in EBNF.a-char, except ".". Space is given a single character encoding, due to its (expected) frequency of use, and backslash as the RFC 822 single quote character.

This mapping is used to transform between the two forms of 822 . word: 822. quoted-string (restricted RFC 822) and 822.atom (restricted RFC 
822). To encode (full RFC 822 -> restricted RFC 822), first remove any quoting from any 822. quoted-string. Then, all EBNF.a-char are used directly and all other CHAR are encoded as EBNF.a-encoded-char.

To decode (restricted RFC $822 \rightarrow$ full RFC 822): if the address can be parsed as EBNF.encoded-atom reverse the previous mapping. If it cannot be so parsed, map the characters directly.

\section{Application}

This mapping should be used for all addresses, at the MTS or Header level. It is applied to the 822.local-part of the addresses. For example:

$\begin{array}{lll}\text { Full RFC } 822 & & \text { Restricted RFC } 822 \\ & & \\ \text { Steve.Kille@cs.ucl.ac.uk } & <-> & \text { Steve.Kille@cs.ucl.ac.uk } \\ \text { "Steve Kille"@cs.ucl.ac.uk } & <-> & \text { Steve_Killedcs.ucl.ac.uk } \\ \text { "argle\#" "@blargle } & <-> & \text { argle\#h\#\#126\#@blargle }\end{array}$

References

[Crocker82a] Crocker, D., "Standard of the Format of ARPA Internet Text Messages", RFC 822, August 1982 .

[Horton86a] Horton, M., "UUCP Mail Interchange Format Standard", RFC 976, February 1986.

[Kille86a] Kille, S., "Mapping Between X.400 and RFC 822", UK Academic Community Report (MG.19), RFC 987, June 1986.

Security Considerations

Security issues are not discussed in this memo.

Author's Address

Steve Kille

University College London

Gower Street

WC1E 6BT

England

Phone: +44-1-380-7294

EMail: S.Kille@Cs.Ucl.AC.UK 